\title{
INTEGRAL OF CURVATURE OF THE NORMAL CONNECTION IN A SUBMANIFOLD OF A RIEMANNIAN MANIFOLD
}

\author{
Dedicated to Professor Shigeru Ishihara on his 60th birthday
}

\author{
By Yosio Mutō
}

$\S 1$. Introduction. Let $\tilde{M}$ and $M$ be orientable $C^{\infty}$ manifolds such that $\operatorname{dim} \tilde{M}=\tilde{m}=m+k$ and $\operatorname{dim} M=m$ and let $f$ be a $C^{\infty}$ embedding of $M$ into $\tilde{M}$. Then there exists a well-known relation between the Pontrjagin classes of $\tilde{M}$, $M$ and the normal bundle $\nu(M)$. This relation is also accessible by the classical method of Riemannian geometry. The purpose of the present paper is to get a formula corresponding to the lowest part of the relation and then to use it to find some properties of an embedded submanifold in some special cases. Indeed our effort is concentrated on integrating the curvature in the normal connection.

We consider that $\tilde{M}$ is endowed with a $C^{\infty}$ Riemannian metric $\tilde{g}$ and $M$ with the Riemannian metric $g$ induced by the embedding. Any 4-cyble in $M$ induces a 4-cycle in $\tilde{M}$ and there exists a relation between the periods on these cycles. In order to get an explicit expression of such a relation we consider a four-dimensional compact orientable $C^{\infty}$ submanifold $N$ of $M$ instead of a 4-cycle. In the beginning part the codimesion $k$ is assumed to be arbitrary but in the last paragraph it is assumed to be 3 .

In $\S 2$ we recall fundamental equations of embedding in Riemannian geometry. The well-known relation of Pontrjagin classes of an embedded manifold is [2], [3], [4]

$$
\begin{aligned}
f^{*}\left(1+P_{4}(\tilde{M})+P_{8}(\tilde{M})+\cdots\right) & \\
=\left(1+P_{4}(M)+P_{8}(M)+\cdots\right)\left(1+P_{4}(\nu(M))+P_{8}(\nu(M))\right. & +\cdots) \\
& \text { mod torsion, }
\end{aligned}
$$

where $\nu(M)$ is the normal bundle. The relation between the periods on a 4cycle $\gamma$ is therefore

$$
\left.f^{*}\left(P_{4}(\tilde{M})\right)\right|_{\gamma}=\left.P_{4}(M)\right|_{\gamma}+\left.P_{4}(\mathcal{\nu}(M))\right|_{\gamma} .
$$

Received June 9, 1981 
The curvature tensor $K_{\mu \lambda P Q}$ of the normal connection plays the central role in $P_{4}(\nu(M))$, but for further treatment a local 3-form $U_{\mu \lambda \kappa}$ which appears in $\S 3$ seems to be more useful. In $\S 4$ two domains are considered in $M$ and the local 3 -forms on them are compared in the intersection. There we find a mapping $\varphi$ of the intersection into $S O(k)$. In $\S 5$ we treat the case $k=3$. In some special case where such mappings $\varphi$ become surjections, we can express the period $\left.P_{4}(\nu(M))\right|_{N}$ by a sum of degrees of mappings $\varphi$. The main results are Theorem 3.1 and Theorem 5.1 .

The author thanks Professor Y. Tomonaga for his kind assistance throughout the work. The author also thanks Professor K. Ogiue whose kind information made the start of the work possible.

\section{§. Fundamental equations of embedding $[1],[5]$.}

Let $(M, g)$ be a Riemannian manifold isometrically embedded in a Riemannian manifold $(\tilde{M}, \tilde{g})$ and let the embedding be expressed locally by

$$
x^{h}=x^{h}\left(y^{1}, \cdots, y^{m}\right), \quad h=1, \cdots, m+k .
$$

We suppose that $\operatorname{dim} M=m, \operatorname{dim} \tilde{M}=\tilde{m}=m+k$ and the letters $x^{h}$ and $y^{\kappa}$ are used as the local coordinates of $\tilde{M}$ and $M$ respectively where the indices run as follows,

$$
h, i, j, \cdots=1, \cdots, m+k ; \quad \kappa, \lambda, \mu, \cdots=1, \cdots, m .
$$

For these indices we adopt usual summation convention. For the indices

$$
P, Q, R, \cdots=m+1, \cdots, m+k
$$

we use the following summation convention,

$$
\sum_{P=m+1}^{m+k} H_{\nu \mu P} H_{\lambda \kappa P} \quad \text { is written } H_{\nu \mu P} H_{\lambda \kappa P} .
$$

We also use the following notations,

$$
\begin{gathered}
B_{\lambda}^{h}=\partial x^{h} / \partial y^{\lambda}=\partial_{\lambda} x^{h}, \quad B_{\mu \lambda}^{i h}=B_{\mu}^{\imath} B_{\lambda}^{h}, \\
g_{\mu \lambda}=B_{\mu \lambda}^{i h} \tilde{g}_{i h}, \\
H_{\mu \lambda}{ }^{h}=\nabla_{\mu} B_{\lambda}^{h}=\partial_{\mu} B_{\lambda}^{h}-\left\{\begin{array}{c}
\kappa \\
\mu \lambda
\end{array}\right\} B_{\kappa}^{h}+\left\{\begin{array}{c}
h \\
j i
\end{array}\right\} B_{\mu \lambda}^{j i},
\end{gathered}
$$

where $\tilde{g}_{i h}$ and $g_{\mu \lambda}$ are respectively the local components of the Riemannian metrics $\tilde{g}$ and $g,\left\{\begin{array}{c}\kappa \\ \mu \lambda\end{array}\right\}$ and $\left\{\begin{array}{c}h \\ j i\end{array}\right\}$ are respectively the Christoffel symbols of $(M, g)$ and $(\tilde{M}, \tilde{g})$ and $H_{\mu \lambda}{ }^{h}$ are the local components of the second fundamental form. We use the symbol $\nabla$ of Riemannian connection in the sense of Van der 
Waerden-Bortolotti with respect to $M$ and $\tilde{M}$ but not with respect to the normal bundle $\nu(M)$.

The curvature tensors, namely the Riemann-Christoffel tensors of $\tilde{M}$ and $M$ are

$$
\begin{aligned}
& \tilde{K}_{k j i}{ }^{h}=\partial_{k}\left\{\begin{array}{c}
h \\
j i
\end{array}\right\}-\partial_{j}\left\{\begin{array}{l}
h \\
k i
\end{array}\right\}+\left\{\begin{array}{l}
h \\
k t
\end{array}\right\}\left\{\begin{array}{c}
t \\
j i
\end{array}\right\}-\left\{\begin{array}{l}
h \\
j t
\end{array}\right\}\left\{\begin{array}{c}
t \\
k i
\end{array}\right\}, \\
& K_{\nu \mu \lambda^{\kappa}}=\partial_{\nu}\left\{\begin{array}{c}
\kappa \\
\mu \lambda
\end{array}\right\}-\partial_{\mu}\left\{\begin{array}{c}
\kappa \\
\nu \lambda
\end{array}\right\}+\left\{\begin{array}{l}
\kappa \\
\nu \tau
\end{array}\right\}\left\{\begin{array}{c}
\tau \\
\mu \lambda
\end{array}\right\}-\left\{\begin{array}{c}
\kappa \\
\mu \tau
\end{array}\right\}\left\{\begin{array}{l}
\tau \\
\nu \lambda
\end{array}\right\} .
\end{aligned}
$$

Now we assume that $O$ is a domain of $M$ such that there exists an orthonormal normal $k$-frame field covering $O$ where the $k$ normal vectors are denoted by $N_{P}, P=m+1, \cdots, m+k$. Denoting their local components by $N_{P}^{h}$ we have

$$
\begin{gathered}
N_{Q}^{\imath} N_{P}^{h} \tilde{g}_{i h}=\delta_{Q P}, \\
\tilde{g}^{j i}=B_{\mu \lambda}^{j i} g^{\mu \lambda}+N_{P}^{\jmath} N_{P}^{i},
\end{gathered}
$$

where $\tilde{g}^{j i}$ and $g^{\mu \lambda}$ are defined by $\tilde{g}_{i t} \tilde{g}^{h t}=\delta_{\imath}^{h}, g_{\lambda \tau} g^{\kappa \tau}=\delta_{\lambda}^{\kappa}$.

From the definition of the symbol $\nabla$ we have

$$
\nabla_{\lambda} N_{P}^{h}=\partial_{\lambda} N_{P}^{h}+B_{\lambda}{ }_{\lambda}\left\{\begin{array}{c}
h \\
j i
\end{array}\right\} N_{P}^{\imath} .
$$

We define as usual $H_{\mu \lambda P}$ and $L_{\lambda P Q}$ by

$$
\begin{gathered}
H_{\mu \lambda P}=H_{\mu \lambda}{ }^{2} N_{P}^{h} \tilde{g}_{i \hbar}, \\
L_{\lambda P Q}=\tilde{g}_{j i}\left(\nabla_{\lambda} N_{P}^{j}\right) N_{Q}^{i}=-L_{\lambda Q P}
\end{gathered}
$$

with the use of which the equations of Gauss, Codazzi and Ricci are written as follows,

where

$$
\begin{gathered}
\tilde{K}_{k j i h} B_{\nu \mu \lambda \kappa}^{k j i h}=K_{\nu \mu \lambda x}-H_{\nu R P} H_{\mu \lambda P}+H_{\mu \kappa P} H_{\nu \lambda P}, \\
\widetilde{K}_{k j i h} B_{\nu \mu \lambda}^{k j i} N_{P}^{h}=\nabla_{\nu} H_{\mu \lambda P}-\nabla_{\mu} H_{\nu \lambda P}-L_{\nu P R} H_{\mu \lambda R}+L_{\mu P R} H_{\nu \lambda R}, \\
\tilde{K}_{k j i h} B_{\nu \mu}^{k j} N_{P} N_{Q}^{h}=\nabla_{\nu} L_{\mu P Q}-\nabla_{\mu} L_{\nu P Q}-L_{\nu P R} L_{\mu R Q}+L_{\mu P R} L_{\nu R Q} \\
+H_{\nu}{ }^{\tau}{ }_{P} H_{\mu \tau Q}-H_{\mu}{ }^{\tau}{ }_{P} H_{\nu \tau Q},
\end{gathered}
$$

$$
\begin{gathered}
\nabla_{\nu} H_{\mu \lambda P}=\partial_{\nu} H_{\mu \lambda P}-\left\{\begin{array}{c}
\tau \\
\nu \mu
\end{array}\right\} H_{\tau \lambda P}-\left\{\begin{array}{c}
\tau \\
\nu \lambda
\end{array}\right\} H_{\mu \tau P}, \\
\nabla_{\mu} L_{\lambda P Q}=\partial_{\mu} L_{\lambda P Q}-\left\{\begin{array}{c}
\tau \\
\mu \lambda
\end{array}\right\} L_{\tau P Q} .
\end{gathered}
$$


When a connection is induced in the normal bundle in the standard way from the Riemannian connections of $\tilde{M}$ and $M$, the resulting curvature tensor is given by

$$
\begin{aligned}
K_{\mu \lambda P Q}= & \nabla_{\mu} L_{\lambda P Q}-\nabla_{\lambda} L_{\mu P Q} \\
& -L_{\mu P R} L_{\lambda R Q}+L_{\lambda P R} L_{\mu R Q} .
\end{aligned}
$$

If we take another $k$-frame field where the normal vectors are ' $N_{P}, P=m+1$, $\cdots, m+k$, then the new $k$-frame field is related to the old one by

$$
{ }^{\prime} N_{P}=a_{P Q} N_{Q},
$$

where the matrix $\left[a_{P Q}\right]$ can be considered as an $S O(k)$ valued function. Corresponding to (2.12) the components of the curvature tensor of the normal connection undergo the transformation

$$
{ }^{\prime} K_{\mu \lambda P Q}=a_{P S} a_{Q T} K_{\mu \lambda S T},
$$

hence

$$
\text { ' } K_{\nu \mu P Q}{ }^{\prime} K_{\lambda \kappa P Q}=K_{\nu \mu P Q} K_{\lambda \kappa P Q} .
$$

This proves that there exists a global 4 -form expressed locally by $K_{\nu \mu P Q} K_{\lambda \kappa P Q}$ $d y^{\nu} d y^{\mu} d y^{2} d y^{\kappa}$.

We get from (2.8) and (2.11)

$$
\tilde{K}_{k j i h} B_{\nu \mu}^{k \jmath} N_{P}^{i} N_{Q}^{h}=K_{\nu \mu P Q}+H_{\nu}{ }^{\tau} P H_{\mu \tau Q}-H_{\mu}{ }^{\tau} P H_{\nu \tau Q} .
$$

\section{$\S 3$. The local 3-form $U_{\mu \lambda \kappa}$.}

In order to find the relation between the Pontrjagin classes $P_{4}(\tilde{M})$ and $P_{4}(M)$ we define the following tensor,

$$
W_{\nu \mu \lambda \kappa}=\tilde{K}_{k j t s} \tilde{K}_{i h m l} \tilde{g}^{t m} \tilde{g}^{s l} B_{\nu \mu \lambda \kappa}^{k j i h} .
$$

For the second member we get in virtue of (2.2)

where

$$
A_{\nu \mu \lambda \kappa}+B_{\nu \mu \lambda \kappa}+C_{\nu \mu \lambda \kappa},
$$

$$
\begin{aligned}
& A_{\nu \mu \lambda \kappa}=\tilde{K}_{k j t s} B_{\nu \mu \tau \sigma}^{k j t s} \tilde{K}_{i \hbar m l} B_{\lambda \kappa \beta \alpha}^{i n m l} g^{\tau \beta} g^{\sigma \alpha}, \\
& B_{\nu \mu \lambda \kappa}=2 \tilde{K}_{k j t s} B_{\nu \mu \tau}^{k j t} N_{P}^{s} \tilde{K}_{i \hbar m l} B_{\lambda \kappa \beta}^{i h m} N_{P}^{l} g^{\tau \beta}, \\
& C_{\nu \mu \lambda \kappa}=\tilde{K}_{k j t s} B_{\nu \mu}^{k j} N_{P}^{t} N_{Q}^{s} \tilde{K}_{i \hbar m l} B_{\lambda \kappa}^{i n} N_{P}^{m} N_{Q}^{l} .
\end{aligned}
$$

Now in view of equations (2.6), (2.7) and (2.14) we get

$$
\begin{aligned}
A_{\nu \mu \lambda \kappa}= & \left(K_{\nu \mu \tau \sigma}-H_{\nu \sigma P} H_{\mu \tau P}+H_{\mu \sigma P} H_{\nu \tau P}\right) \\
& \times\left(K_{\lambda \kappa \beta \alpha}-H_{\lambda \alpha Q} H_{\kappa \beta Q}+H_{\kappa \alpha Q} H_{\lambda \beta Q}\right) g^{\tau \beta} g^{\sigma \alpha},
\end{aligned}
$$




$$
\begin{aligned}
B_{\nu \mu \lambda \kappa}= & 2\left(\nabla_{\nu} H_{\mu \tau P}-\nabla_{\mu} H_{\nu \tau P}-L_{\nu P Q} H_{\mu \tau Q}+L_{\mu P Q} H_{\nu \tau Q}\right) \\
& \times\left(\nabla_{\lambda} H_{\kappa \beta P}-\nabla_{\kappa} H_{\lambda \beta P}-L_{\lambda P R} H_{\kappa \beta R}+L_{\kappa P R} H_{\lambda \beta R}\right) g^{\tau \beta}, \\
C_{\nu \mu \lambda \kappa}= & \left(K_{\nu \mu P Q}+H_{\nu}{ }^{\beta}{ }_{P} H_{\mu \beta Q}-H_{\mu}{ }^{\beta}{ }_{P} H_{\nu \beta Q}\right) \\
& \times\left(K_{\lambda \kappa P Q}+H_{\lambda}{ }^{\alpha}{ }_{P} H_{\kappa \alpha Q}-H_{\kappa}{ }^{\alpha}{ }_{P} H_{\lambda a Q}\right)
\end{aligned}
$$

and, as we need only the skew symmetric part $S_{\nu \mu \lambda_{k}}=W_{\left[\nu \mu \lambda_{k}\right]}$ of $W_{\nu \mu \lambda k}$, we can write

$$
\begin{aligned}
& A_{\nu \mu \lambda \kappa} \sim K_{\nu \mu \tau \sigma} K_{\lambda \kappa}{ }^{\tau \sigma}-4 K_{\nu \mu \tau \sigma} H_{\lambda}{ }^{\sigma}{ }_{P} H_{\kappa}{ }^{\tau} P+4 H_{\nu \sigma P} H_{\mu \tau P} H_{\lambda}{ }^{\sigma}{ }_{Q} H_{\kappa}{ }^{\tau} Q, \\
& B_{\nu \mu \lambda \kappa} \sim 8 \nabla_{\nu} H_{\mu \tau P} \nabla_{\lambda} H_{\kappa}{ }^{\tau} P-16 \nabla_{\nu} H_{\mu \tau P} L_{\lambda P Q} H_{\kappa}^{\tau} Q+8 L_{\nu P Q} H_{\mu \tau Q} L_{\lambda P R} H_{\kappa}^{\tau} R \text {, } \\
& C_{\nu \mu \lambda \kappa} \sim K_{\nu \mu P Q} K_{\lambda \kappa P Q}+4 K_{\nu \mu P Q} H_{\lambda}{ }^{\alpha}{ }_{P} H_{\kappa \alpha Q}+4 H_{\nu}{ }^{\beta}{ }_{P} H_{\mu \beta Q} H_{\lambda}{ }^{\alpha}{ }_{P} H_{\kappa \alpha Q} \\
& \sim K_{\nu \mu P Q} K_{\lambda \kappa P Q}+8 \nabla_{\nu} L_{\mu P Q} H_{\lambda}{ }^{\alpha}{ }_{P} H_{\kappa \alpha Q} \\
& -8 L_{\nu P R} L_{\mu R Q} H_{\lambda}{ }^{\alpha}{ }_{P} H_{\kappa \alpha Q}+4 H_{\nu}{ }^{\beta}{ }_{P} H_{\mu \beta Q} H_{\lambda}{ }^{\alpha}{ }_{P} H_{\kappa \alpha Q},
\end{aligned}
$$

where $\sim$ means equality of the skew symmetric parts.

As we have

$$
\begin{aligned}
& 8 \nabla_{\nu} H_{\mu \tau P} \nabla_{\lambda} H_{\kappa}{ }^{\tau} P \\
& \sim 8 \nabla_{\nu}\left(H_{\mu \tau P} \nabla_{\lambda} H_{\kappa}{ }^{\tau} P\right)-4 H_{\mu \tau P}\left(\nabla_{\nu} \nabla_{\lambda} H_{\kappa}{ }^{\tau} P-\nabla_{\lambda} \nabla_{\nu} H_{\kappa}{ }^{\tau} P\right) \\
& \sim 4 \nabla_{\nu}\left(H_{\mu}{ }^{\tau} P \tilde{K}_{k j i \hbar} B_{\lambda \kappa \tau}^{k j \imath} N_{P}^{h}+2 H_{\mu}{ }^{\tau} P L_{\lambda P Q} H_{\kappa \tau Q}\right) \\
&-4 H_{\mu \tau P} K_{\nu \lambda \sigma}{ }^{\tau} H_{\kappa}{ }^{\sigma} P \\
&-16 \nabla_{\nu} H_{\mu \tau P} L_{\lambda P Q} H_{\kappa}{ }^{\tau}{ }+8 \nabla_{\nu} L_{\mu P Q} H_{\lambda}{ }^{\alpha}{ }_{P} H_{\kappa \alpha Q} \\
& \sim 8 \nabla_{\nu}\left(L_{\mu P Q} H_{\lambda}{ }^{\alpha}{ }_{P} H_{\kappa \alpha Q}\right) \\
& \sim-8 \nabla_{\nu}\left(H_{\mu}{ }^{\tau}{ } L_{\lambda P Q} H_{\kappa \tau Q}\right),
\end{aligned}
$$

we get after some calculation

or

$$
S_{\nu \mu \lambda \kappa} \sim K_{\nu \mu \tau \sigma} K_{\lambda \kappa}{ }^{\tau \sigma}+4 \nabla_{\nu}\left(H_{\mu}{ }^{\tau}{ }^{\tau} \tilde{K}_{k \jmath i h} B_{\lambda \kappa \tau}^{k \jmath \imath}\right)+K_{\nu \mu P Q} K_{\lambda \kappa P Q}
$$

$$
\tilde{K}_{k j t}{ }^{s} \tilde{K}_{i h s^{t}} B_{\nu \mu \lambda k}^{k j i h} \sim K_{\nu \mu \tau}{ }^{\sigma} K_{\lambda \kappa \sigma}{ }^{\tau}-4 \nabla_{\nu}\left(H_{\mu}{ }^{\tau}{ }^{h} \widetilde{K}_{k j i h} B_{\lambda \kappa \tau}^{k j \downarrow}\right)-K_{\nu \mu P Q} K_{\lambda \kappa P Q},
$$

which is an equation globally valid.

From (3.2) we see that for any 4-cycle $\gamma$ of $M$ we have

$$
\left.P_{4}(\nu(M))\right|_{\gamma}=\alpha_{1} \int_{\gamma} K_{\nu \mu P Q} K_{\lambda \kappa P Q} d y^{\nu} d y^{\prime \prime} d y^{\lambda} d y^{\kappa}
$$

for the period on $\gamma$ of the Pontrjagin class $P_{4}$ of the normal bundle.

From (2.11) we get 


$$
\begin{aligned}
K_{\nu / \mu P Q} K_{\lambda \kappa P Q} \sim & 4 \nabla_{\nu} L_{\mu P Q} \nabla_{\lambda} L_{\kappa P Q}-8 \nabla_{\nu} L_{\mu P Q} L_{\lambda P R} L_{\kappa R Q} \\
& +4 L_{\nu P R} L_{\mu R Q} L_{\lambda P S} L_{\kappa S Q} \\
& \sim 4 \nabla_{\nu}\left(L_{\mu P Q} \nabla_{\lambda} L_{\kappa P Q}-(2 / 3) L_{\mu P Q} L_{\lambda P R} L_{\kappa R Q}\right)
\end{aligned}
$$

as we have $L_{\nu P R} L_{\mu R Q} L_{\lambda P S} L_{\kappa S Q} \sim 0$ in view of

$$
\begin{aligned}
& L_{\nu P R} L_{\mu R Q} L_{\lambda P S} L_{\kappa S Q}=L_{\nu R P} L_{\mu P Q} L_{\lambda R S} L_{\kappa S Q} \\
& \quad=L_{\nu R P} L_{\lambda R S} L_{\mu P Q} L_{\kappa S Q}=L_{\nu R P} L_{\lambda R Q} L_{\mu P S} L_{\kappa Q S} \\
& \quad=L_{\nu P R} L_{\lambda R Q} L_{\mu P S} L_{\kappa S Q} \sim-L_{\nu P R} L_{\mu R Q} L_{\lambda P S} L_{\kappa S Q} .
\end{aligned}
$$

Thus we get

$$
K_{\nu \mu P Q} K_{\lambda \kappa P Q} \sim \nabla_{\nu} U_{\mu \lambda \kappa},
$$

where $U_{\mu \lambda x}$ is a skew symmetric tensor defined by

$$
U_{\mu \lambda \kappa} \sim 4 L_{\mu P Q} \nabla_{\lambda} L_{\kappa P Q}-(8 / 3) L_{\mu P Q} L_{\lambda P R} L_{\kappa R Q} \text {. }
$$

As this tensor is defined only in the domain $O$, the closed form

is not a derived form.

$$
K_{\nu \mu P Q} K_{\lambda k P Q} d y^{\nu} d y^{\mu} d y^{\lambda} d y^{\kappa}
$$

Thus we have proved the following theorem.

Theorem 3.1. The Pontrjagin class $P_{4}(\nu(M))$ of the normal bundle $\nu(M)$ is represented by the closed form

$$
\alpha_{1} K_{\nu \mu Q Q} K_{\lambda \kappa P Q} d y^{\nu} d y^{\mu} d y^{\lambda} d y^{\kappa},
$$

where $K_{\mu \lambda P Q}$ is the curvature tensor of the normal connection. This Pontrjagin class is locally represented by the derived 4-form

$$
4 \alpha_{1} \nabla_{\nu}\left(L_{\mu P Q} \nabla_{\lambda} L_{\kappa P Q}-(2 / 3) L_{\mu P Q} L_{\lambda P R} L_{\kappa R Q}\right) d y^{\nu} d y^{\mu} d y^{\lambda} d y^{\kappa},
$$

where locally means in each open set where an orthonormal normal k-frame field exists.

\section{$\S 4$. The relation between local 3-forms in the overlapping domain.}

In the foregoing paragraph we considered only one domain $O$ in $M$. Now we assume that $O$ and ' $O$ are domains over each of which there exists an orthonormal normal $k$-frame field and $O \cap^{\prime} O \neq \emptyset$. We denote by $\left\{N_{P}\right\}$ the $k$ frame field on $O$ and by $\left\{{ }^{\prime} N_{P}\right\}$ the $k$-frame field on ' $O$. Then in $O \cap^{\prime} O$ we have

$$
{ }^{\prime} N_{P}=a_{P S} N_{S},
$$

where the $k \times k$ matrix $\left[a_{P S}\right]$ is an orthogonal matrix with $\operatorname{det}\left[a_{P S}\right]=1$. We want to find the relation between the 3 -forms $U_{\mu \lambda_{k}}$ and ' $U_{\mu \lambda_{k}}$ obtained respec- 
tively on $O$ and ' $O$.

In view of (2.5) and (4.1) we get

$$
{ }^{\prime} L_{\mu P Q}=a_{Q T} a_{P S} L_{\mu S T}+a_{Q S} \partial_{\mu} a_{P S}
$$

and

$$
\begin{aligned}
\nabla_{\lambda}^{\prime} L_{\kappa P Q} \sim a_{Q T} a_{P S} \nabla_{\lambda} L_{\kappa S T}+\left(\partial_{\lambda} a_{Q T} a_{P S}+a_{Q T} \partial_{\lambda} a_{P S}\right) L_{\kappa S T} \\
+\partial_{\lambda} a_{Q S} \partial_{\kappa} a_{P S},
\end{aligned}
$$

where the sign $\sim$ is used instead of the equality sign when only the skew symmetric parts with respect to Greek indices are considered. From (4.2) and (4.3) we get after straightforward calculation

hence

$$
\begin{aligned}
\left(3^{\prime} L_{\mu P Q} \nabla_{\lambda}{ }^{\prime} L_{\kappa P Q}-2^{\prime} L_{\mu P Q} L_{\lambda P R}{ }^{\prime} L_{\kappa R Q}\right) \\
\quad-\left(3 L_{\mu P Q} \nabla_{\lambda} L_{\kappa P Q}-2 L_{\mu P Q} L_{\lambda P R} L_{\kappa R Q}\right) \\
\quad \sim 3 \nabla_{\lambda}\left(a_{P T} \partial_{\mu} a_{P S} L_{\kappa T S}\right)-a_{Q T} \partial_{\mu} a_{Q S} \partial_{\lambda} a_{P T} \partial_{\kappa} a_{P S},
\end{aligned}
$$

$$
{ }^{\prime} U_{\mu \lambda \kappa}-U_{\mu \lambda \kappa} \sim 4 \nabla_{\mu}\left(a_{P T} \partial_{\lambda} a_{P S} L_{\kappa S T}\right)-(4 / 3) a_{Q T} \partial_{\mu} a_{Q S} \partial_{\lambda} a_{P T} \partial_{\kappa} a_{P S} .
$$

We have

$$
\begin{aligned}
& \partial_{\nu} a_{L T} \partial_{\mu} a_{L S} \partial_{\lambda} a_{K T} \partial_{\kappa} a_{K S} \\
& \quad=\left(a_{Q V} \partial_{\nu} a_{Q T}\right)\left(a_{L V} \partial_{\mu} a_{L S}\right)\left(a_{P U} \partial_{\lambda} a_{P T}\right)\left(a_{K U} \partial_{\kappa} a_{K S}\right) .
\end{aligned}
$$

If we define $A_{\mu T S}$ by $A_{\mu T S}=a_{P T} \partial_{\mu} a_{P S}$, then we have $A_{\mu T S}=-A_{\mu S T}$ and

$$
\begin{aligned}
& A_{\nu V T} A_{\mu V S} A_{\lambda U T} A_{\kappa U S}=A_{\nu T V} A_{\mu T S} A_{\lambda U V} A_{\kappa U S} \\
& =A_{\nu T V} A_{\lambda U V} A_{\mu T S} A_{\kappa U S}=A_{\nu T V} A_{\lambda S V} A_{\mu T U} A_{\kappa S U} \\
& =A_{\nu V T} A_{\lambda V S} A_{\mu U T} A_{\kappa U S} \sim-A_{\nu V T} A_{\mu V S} A_{\lambda U T} A_{\kappa U S} .
\end{aligned}
$$

This proves that

$$
a_{Q T} \partial_{\mu} a_{Q S} \partial_{\lambda} a_{P T} \partial_{\kappa} a_{P S} d y^{\mu} d y^{\lambda} d y^{\kappa}
$$

is a closed 3-form. We also have

$$
\begin{aligned}
& a_{Q T} \partial_{\mu} a_{Q S} \partial_{\lambda} a_{P T} \partial_{\kappa} a_{P S} \\
& \quad=\left(a_{R T} \partial_{\mu} a_{R S}\right)\left(a_{Q U} \partial_{\lambda} a_{Q T}\right)\left(a_{P U} \partial_{\kappa} a_{P S}\right)
\end{aligned}
$$

and (4.5) is the pull back $\varphi^{*} \omega$ of a closed 3-form $\omega$ on $S O(k)$ where $\varphi$ is a mapping $\varphi: O \cap^{\prime} O \rightarrow S O(k)$.

Let us consider that a compact orientable four-dimensional manifold $N$ is embedded in $M$ and assume that $N$ can be covered by domains $O_{1}, \cdots, O_{A}$ of 
$M$ such that for each $a=1, \cdots, A$ there exists an orthonormal normal $k$-frame field on $O_{a}$ and such that $O_{a} \cap O_{b} \cap O_{c} \cap N=\emptyset$ for $1 \leqq a<b<c \leqq A$. If $D_{1}, \cdots, D_{A}$ are domains of $N$ such that $D_{a} \cap D_{b}=\emptyset$ for $1 \leqq a<b \leqq A, \bar{D}_{a} \subset O_{a}$ for $1 \leqq a \leqq A$ where $\bar{D}_{a}$ is the closure of $D_{a}, N$ is covered by $\bar{D}_{1}, \cdots, \bar{D}_{A}$ and furthermore, $\bar{D}_{a} \cap \bar{D}_{b}=D_{a, b}$ are closed $C^{\infty}$ submanifolds of $N$ or $\emptyset$, then we have in view of (3.4)

$$
\begin{aligned}
& \int_{N} K_{\nu / \ell P Q} K_{\lambda \kappa P Q} d y^{\nu} d y^{\mu} d y^{\lambda} d y^{\kappa} \\
& \quad=\sum_{a=1}^{A} \int_{D_{a}} \nabla_{\nu} U_{\mu \lambda \kappa} d y^{\nu} d y^{\mu} d y^{\lambda} d y^{\kappa} \\
& \quad=\sum_{1 \leqq a<b \leqq A} \int_{D_{a, b}}\left(U_{\mu \lambda \kappa}^{(a)}-U_{\mu \lambda \kappa}^{(b)}\right) d y^{\mu} d y^{\lambda} d y^{\kappa},
\end{aligned}
$$

where $D_{a, b}$ is endowed with a suitable orientation. Then we have a mapping $D_{a, b} \rightarrow S O(k)$ as the restriction of $\varphi_{a, b}: O_{a} \cap O_{b} \rightarrow S O(k)$ which we also write $\varphi_{a, b}: D_{a, b} \rightarrow S O(k)$. In view of (4.4) we get

$$
\begin{gathered}
\int_{N} K_{\nu \mu P Q} K_{\lambda \kappa P Q} d y^{\nu} d y^{\mu} d y^{\lambda} d y^{\kappa}=-\sum_{1 \leqq a<b \leqq A} I_{a, b}, \\
I_{a, b}=\frac{4}{3} \int_{D_{a, b}} \varphi_{a, b} * \omega .
\end{gathered}
$$

If we take local coordinates $u^{\alpha}, \alpha=1, \cdots, k(k-1) / 2$, in $S O(k)$ and put $R=\left[a_{P S}\right]$, the closed 3 -form $\omega$ is given by

$$
\omega=\operatorname{trace}\left(\left(R^{-1} \partial_{\gamma} R\right)\left(R^{-1} \partial_{\beta} R\right)\left(R^{-1} \partial_{\alpha} R\right)\right) d u^{\gamma} d u^{\beta} d u^{\alpha},
$$

where $\partial_{\alpha}=\partial / \partial u^{\alpha}$.

\section{$\S 5$. Some special cases with $k=3$.}

If $k=3, \omega$ takes a simple form. In this case we can take as $R$ a matrix with entries

$$
\begin{aligned}
& R_{11}=\cos \varphi \cos \theta \cos \psi-\sin \varphi \sin \psi, \\
& R_{12}=\sin \varphi \cos \theta \cos \psi+\cos \varphi \sin \psi, \\
& R_{13}=-\sin \theta \cos \psi, \\
& R_{2 \alpha}=\partial R_{1 \alpha} / \partial \psi, \quad \alpha=1,2,3, \\
& R_{31}=\cos \varphi \sin \theta, \quad R_{32}=\sin \varphi \sin \theta, \quad R_{33}=\cos \theta .
\end{aligned}
$$

Then we have 


$$
\begin{aligned}
R^{-1} \partial_{1} R & =\left(\begin{array}{ccc}
0 & 0 & -\cos \varphi \\
0 & 0 & -\sin \varphi \\
\cos \varphi & \sin \varphi & 0
\end{array}\right), \\
R^{-1} \partial_{2} R & =\left(\begin{array}{ccc}
0 & 1 & 0 \\
-1 & 0 & 0 \\
0 & 0 & 0
\end{array}\right), \\
R^{-1} \partial_{3} R & =\left(\begin{array}{ccc}
0 & \cos \theta & -\sin \theta \sin \varphi \\
-\cos \theta & 0 & \sin \theta \cos \varphi \\
\sin \theta \sin \varphi & -\sin \theta \cos \varphi & 0
\end{array}\right),
\end{aligned}
$$

where we have put $u^{1}=\theta, u^{2}=\varphi, u^{3}=\phi$. From this we get

$$
\begin{aligned}
& \left\{\left(R^{-1} \partial_{1} R\right)\left(R^{-1} \partial_{2} R\right)-\left(R^{-1} \partial_{2} R\right)\left(R^{-1} \partial_{1} R\right)\right\}\left(R^{-1} \partial_{3} R\right) \\
& \quad=\left(\begin{array}{ccc}
\sin \theta \sin ^{2} \varphi & -\sin \theta \cos \varphi \sin \varphi & 0 \\
-\sin \theta \cos \varphi \sin \varphi & \sin \theta \cos ^{2} \varphi & 0 \\
-\cos \theta \cos \varphi & -\cos \theta \sin \varphi & \sin \theta
\end{array}\right),
\end{aligned}
$$

hence

$$
\omega=6 \sin \theta d \theta d \varphi d \psi .
$$

As the degree $n_{a, b}$ of the mapping $\varphi_{a, b}: D_{a, b} \rightarrow S O(3)$ is given by

$$
\int_{D_{a, b}} \varphi_{a, b} * \omega=n_{a, b} \int_{S O(3)} \omega
$$

we get in view of (5.1)

$$
\int_{D_{a, b}} \varphi_{a, b} * \omega=48 \pi^{2} n_{a, b}
$$

Hence we have from (4.7)

$$
I_{a, b}=64 \pi^{2} n_{a, b} .
$$

This proves in virtue of (3.3), (4.6) and (4.7) the following theorem.

THEOREM 5.1. Let $(M, g)$ be an orientable Riemannian manifold of dimension $m$ isometrically embedded in an orientable Riemannian manifold $(\tilde{M}, \tilde{g})$ of dimension $m+3$. The normal bundle is denoted by $\nu(M)$. Let $N$ be a four-dimensional compact orientable submanifold embedded in $M$. We assume that there exist domains $O_{1}, \cdots, O_{A}$ of $M$ satisfying $O_{1} \cup \cdots \cup O_{A} \supset N$, admitting for each $a=1$, ․, A an orthonormal normal 3-frame field of $\nu(M)$ on $O_{a}$ and such that $O_{a} \cap$ 
$O_{b} \cap O_{c} \cap N=\emptyset$ for $1 \leqq a<b<c \leqq A$. Let $D_{1}, \cdots, D_{A}$ be domains of $N$ satisfying $D_{a} \cap D_{b}=\emptyset$ for $1 \leqq a<b \leqq A, \bar{D}_{a} \subset O_{a}$ for $1 \leqq a \leqq A, N=\bar{D}_{1} \cup \cdots \cup \bar{D}_{A}$ and such that $\bar{D}_{a} \cap \bar{D}_{b}=D_{a, b}$ are closed $C^{\infty}$ submanifolds of $N$ or $\emptyset$. Then we have for the period of $P_{4}(\nu(M))$ on $N$

$$
\left.P_{4}(\nu(M))\right|_{N}=-64 \alpha_{1} \pi^{2} n,
$$

where $n$ is the sum of the mapping degree $n_{a, b}$ of the mapping $\varphi_{a, b}: D_{a, b} \rightarrow S O(3)$.

We can consider the case where $M$ coincides with $N$.

We have as yet no positive example. The following example is a negative one.

$P^{2}(C)$ can not be embedded in $R^{7}$ in such a way as stated in Theorem 5.1.

Proof. If $M=N=P^{2}(C)$ in $R^{7}$ satisfies the condition stated above, we get $P_{4}\left[P^{2}(C)\right]=-\left.P_{4}(\nu(M))\right|_{N}=64 \alpha_{1} \pi^{2} n$. But it is known that [3]

$$
P_{4}\left[P^{2}(C)\right]=96 \alpha_{1} \pi^{2} \text {. }
$$

\section{REFERENCES}

[1] Chen, B.-Y., Geometry of Submanifolds, Marcel Dekker, New York, 1973.

[2] Hirzebruch, F., Topological Methods in Algebraic Geometry, Springer, Berlin, Heidelberg, New York, 1978.

[3] Milnor, J.W. And J.D. Stasheff, Characterıstic Classes, Annals of Math. Studies 76, Princeton, 1974.

[4] Tomonaga, Y., Differential Geometry in the Large (in Japanese), Mimeographed Notes, Utsunomiya University, 1967.

[5] YANO, K., Introduction to Riemannian Geometry (in Japanese), Morikita, 1971.

Musashi Institute of Technology

Tamazutsumi, Setagaya-ku,

TOKYO 158,

2262-150, TоміокA-сHO,

Kanazawa-ku, Yokohama 236,

JAPAN 